\title{
Small dose of ephedrine for prevention of hypotension following propofol and fentanyl administration during induction of general anesthesia
}

\author{
Gamal Ejaimi, ${ }^{1}$ Abla Saab, ${ }^{2}$ Sittelnissa Ahmed, ${ }^{3}$ Areeg Ahmed, ${ }^{4}$ Hussain Abujamilah, ${ }^{5}$ \\ Bhanuprakash Satyanarayana ${ }^{1}$ \\ ${ }^{1}$ Department of Anesthesia and Intensive Care, Taiba Hospital, Kuwait; ${ }^{2}$ Department of Anesthesia \\ and Intensive Care, College of Medicine, Ain Shams University, Cairo, Egypt; ${ }^{3}$ Department of \\ Intensive Care, Aseer Central Hospital, Abha, Saudi Arabia; ${ }^{4}$ Department of Anesthesia and Intensive \\ Care, Soba University Hospital, Khartoum, Sudan; ${ }^{5}$ Department of Anesthesia and Pain Management, \\ Aseer Central Hospital, Abha, Saudi Arabia
}

\begin{abstract}
Induction of general anesthesia with propofol and fentanyl could result in hypotension and bradycardia. Various methods are being used to prevent these adverse effects. The aim of our study was to assess the efficacy and safety of a small dose of ephedrine in prevention of hypotension following administration of propofol
\end{abstract}

\begin{abstract}
Correspondence: Gamal Abdalla Mohamed Ejaimi, Department of Anaesthesia and Intensive care, Taiba Hospital, Kuwait.

Tel.: +965.95580069.

E-mail; gamalejaimi@hotmail.com

Key words: Ephedrine; bradycardia; hypotension; fentanyl; propofol.

Contributions: GE: conceptualization, literature review, study design, data collection, data analysis, discussion, manuscript writing and final authorization; AS: literature review, discussion, manuscript writing and final authorization; SA: conceptualization, literature review, study design, data collection, data analysis, discussion, manuscript writing and final authorization; AA: conceptualization, literature review, discussion, and manuscript writing; HA: conceptualization, literature review, study design, discussion, and manuscript writing; BS: discussion and manuscript writing.
\end{abstract}

Conflict of interest: The authors have no conflict of interest to declare.

Acknowledgments: We are grateful to our colleagues in anaesthesia department of Aseer Central Hospital, Abha, KSA.

Ethics approval and consent to participate: Approval Number No. 20160302 from Aseer Central Hospital (ACH) Ethics and IRB (lnternal Review Board) Committee.

Received for publication: 9 June 2020 .

Revision received: 26 January 2020.

Accepted for publication: 30 Janaury 2020.

This work is licensed under a Creative Commons Attribution NonCommercial 4.0 License (CC BY-NC 4.0).

(C) Copyright: the Author(s),2020

Licensee PAGEPress, Italy

Annals of African Medical Research 2020; 3:126

doi:10.4081/aamr.2020.126 and fentanyl. This prospective, randomized, comparative study was conducted among a total of 50 patients, both genders, age 18 years up to 60 years with ASA grade I and II and some of class III, presented for elective surgery under general anesthesia. Patients were randomly allocated into one of two groups (25 patients in each): $\mathrm{CG}$ (the control group), which received propofol in a dose of $2 \mathrm{mg} / \mathrm{kg}$, intravenously, over 20-30s mixed with $2 \mathrm{~mL}$ normal saline: and EphG (Ephedrine group), while received propofol in a dose of $2 \mathrm{mg} / \mathrm{kg}$, intravenously, over 20-30s mixed with $2 \mathrm{~mL}$ of ephedrine $(10 \mathrm{mg})$. The Mean Arterial Blood Pressure (MAP) and Heart Rate (HR) were recorded before induction and then every 1 min up to $6 \mathrm{~min}$ after induction. The categorical data are presented as a number and percentage and were subjected to Fisher's exact or Chi-square test for analysis. The statistical significance was $\mathrm{p} \leq 0.05$. The significant differences in HR were observed in the $3^{\text {rd }}, 4^{\text {th }}$, and $5^{\text {th }}$ minutes with P-value, $0.018,0.000,0.000$, respectively. However, no patient in the study participants had bradycardia. The significant differences in MAP were observed in the $2^{\text {nd }}$, $3^{\text {rd }}, 4^{\text {th }}$, and $5^{\text {th }}$ minutes with P-value, $0.035,0.000,0.000$, and 0.000 , respectively. The percentage of patient in CG who developed hypotension in the $3^{\text {rd }}$ and $4^{\text {th }}$ is $44 \%$ and $32 \%$ compared to $8 \%$ and $0 \%$ in EphG, with significant differences (P-value 0.004 and 0.002 , respectively). Administration of small dose of ephedrine with propofol could attenuate propofol/fentanyl hypotensive and bradycardic effects.

\section{Introduction}

Propofol, which is an intravenous anesthetic agent, nowadays, is commonly used as an induction agent of choice during general anesthesia. It has a rapid action and rapid recovery with decreased hangover effect. ${ }^{1,2}$ The problem of propofol is a sudden drop in arterial pressure. This hypotensive effect of propofol can be enhanced when combined with fentanyl even in well-rehydrated patients. $^{2}$ Fentanyl, which is a short-acting opioid, is a very potent analgesic and can cause hypotension and bradycardia alone. This hypotensive effect of the combination may lead to very low blood pressure and even impalpable pulse. ${ }^{2}$

In some studies, various methods are being used such as a combination of propofol with ketamine, ephedrine, atropine, glycopyrrolate, dopamine, dobutamine, and metaraminol in the prevention of this hypotension. ${ }^{3}$ Ephedrine has a sympathomimetic effect resulting in the stimulation of both alpha- and beta-adrenergic receptors and also releases noradrenaline from the storage site. 
Ephedrine also can be used for relaxation of bronchial smooth muscle, cardiac stimulation, and increased systolic and usually diastolic blood pressure via an increase in cardiac output and peripheral vasoconstriction. ${ }^{4}$ Ephedrine also causes a decrease in intestinal tone and motility, relaxation of the bladder wall, contraction of the sphincter muscle, relaxation of the detrusor muscle, and a decrease in the uterine activity. ${ }^{4}$ It was hypothesized that a small dose of ephedrine, combined with propofol could decrease the hypotensive effect of propofol and fentanyl during the induction of general anesthesia. In our study, we have aimed to assess the efficacy and safety of a small dose of ephedrine in the prevention of hypotension following administration of propofol and fentanyl during induction of general anesthesia. The secondary objective was to assess propofol-fentanyl-related complications.

\section{Materials and Methods}

This double-blind, randomized, controlled trial study was conducted in Aseer Central Hospital (ACH), Abha, KSA, from January 2017 to July 2017, following obtaining the Ethical approval from ACH Ethics and Internal Review Board (IBR) committee. Informed written consent was obtained. A total of 50 patients, both genders, age 18 years up to 60 years with ASA grade I and II and some of class III, presented for elective surgery under general anesthesia, were enrolled. Patients with a history of closedangle glaucoma, pregnancy, pheochromocytoma, asymmetric septal hypertrophy, idiopathic hypertrophic subaortic stenosis, Monoamine Oxidase Inhibitor (MAOIs), hypersensitivity to ephedrine, contraindication to propofol, and hypotension or shocked were excluded from the study as well as patient unwilling to participate in the study.

Following preoxygenation for 3 to $5 \mathrm{~min}$, induction with propofol, fentanyl, and rocuronium was carried out. Patients were randomly allocated into one of two groups (25 patients in each): CG (the control group), which received propofol in a dose of 2 $\mathrm{mg} / \mathrm{kg}$, intravenously, over 20-30 s mixed with $2 \mathrm{~mL}$ normal saline: and EphG (Ephedrine group), which received propofol in a dose of $2 \mathrm{mg} / \mathrm{kg}$, intravenously, over 20-30 s mixed with $2 \mathrm{ml}$ of ephedrine (10 mg). The Mean Arterial Blood Pressure (MAP) and Heart Rate (HR) were recorded before induction and then every 1 min up to 6 min after induction. Ringer's lactate solution was used for the maintenance and replacement fluids. Drugs were prepared and administered by an assigned nurse of anesthesia. Drugs were blinded from assigned anesthesia doctor and monitoring anesthesia nurse.

Recording of HR, Systolic Blood Pressure (SBP), Diastolic Blood Pressure (DBP), and mean arterial blood pressure were carried out just before induction and every minute after induction up to 6 minutes. Hypotension was defined when there was a drop-in MAP of at least $20 \%$ from the baseline. Other monitoring of the vital signs of patients was continued as usual. The primary outcome of the study was the attenuation of the hypotensive effect of propofol/fentanyl by a small dose of ephedrine. The secondary objectives were the adverse effect of ephedrine. For data entry and analysis, the Statistical Package for Social Sciences 21.0 (IBM, Armonk, New York, USA) was used. For sample size calculation, the MAP was used as the primary outcome of this study. To achieve a 2 -sided type 1 error of $0.05 \%$ with a power of $80 \%$, a total number of 50 patients, 25 in each group, would be enough. A number of 54 patients were enrolled in the study and due to the exclusion of 4 patients, 50 patients were included in the study, 25 patients in each group (Figure 1). The categorical data are present- ed as a number and percentage and were subjected to Fisher's exact or Chi-square test for analysis. The continuous data are presented as mean and standard deviation and were subjected to student t. test for analysis. The statistical significance was considered at $\mathrm{p} \leq 0.05$.

\section{Results}

A total of 50 patients were included in this study. The demographic of the study patients, in the two groups, in respect to age, gender, weight, and ASA grade were compared with no statistical differences (Table 1).

Two groups show no differences in the base heart rate. The mean of the heart rate in $\mathrm{CG}$ in the first and second minutes show no difference between the two groups. The significant differences were observed in the $3^{\text {rd }}, 4^{\text {th }}$, and $5^{\text {th }}$ minutes with P-value, 0.18 , $0.000,0.000$, respectively (Table 2). However, no patient in the study participants had bradycardia.

Table 1. The characteristic demographic between the two groups.

\begin{tabular}{lccc} 
Character & $\mathrm{CG}, \mathrm{n}=25(\%)$ & EphG, $\mathrm{n}=25(\%)$ & P-value \\
Age $40+4.7$ & $39.6+5.6$ & 0.7856 & \\
Weight & $75.32+4.3$ & $74.86+3.9$ & 0.6937 \\
\hline $\begin{array}{l}\text { Gender } \\
\quad \text { Male }\end{array}$ & $13(52)$ & $11(44)$ & 0.7880 \\
$\quad$ Female & $12(48)$ & $14(66)$ & \\
ASA & $15(60)$ & $15(60)$ & 0.821 \\
$\quad$ I & $8(32)$ & $9(36)$ & \\
II & $2(8)$ & $1(4)$ & \\
III & & & \\
\hline
\end{tabular}

$\mathrm{CG}=$ Control Group. $\mathrm{EphG}=$ Ephedrine

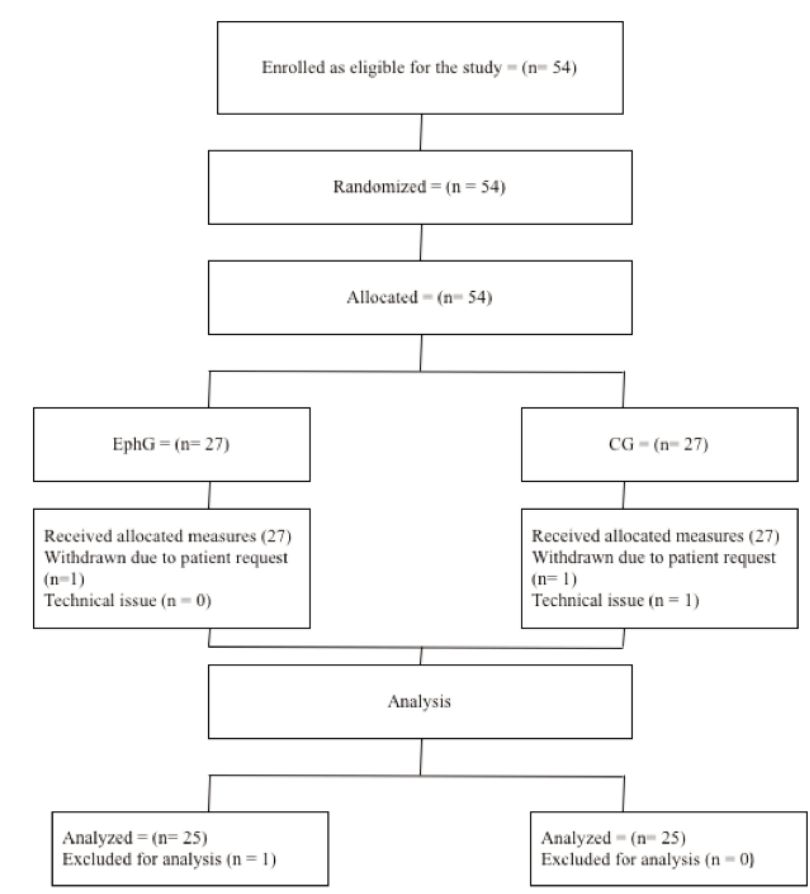

Figure 1. Diagram for sample size. 
Two groups show no differences in the base MAP. The mean of the heart rate in $\mathrm{CG}$ in the first minute shows no difference between the two groups. The significant differences were observed in the $2^{\text {nd }}, 3^{\text {rd }}, 4^{\text {th }}$, and $5^{\text {th }}$ minutes with P-value, 0.035, 0.000, 0.000 , and 0.000 , respectively (Table 3 ).

No patient developed hypertension in the study groups. During the $1^{\text {st }}$ minute no patient had hypotension. In the second minute $12 \%$ of patient in the CG group developed hypotension compared to zero patient in EphG with no statistical difference (P-value 0.07). The percentage of patient in CG who developed hypotension in the $3^{\text {rd }}$ and $4^{\text {th }}$ is $44 \%$ and $32 \%$ compared to $8 \%$ and $0 \%$ with significant differences with a p-value 0.004 and 0.002 , respectively. No patient had hypotension in the $5^{\text {th }}$ minute (Table 4 ).

\section{Discussion}

Induction of general anesthesia using propofol and fentanyl could result in hypotension. ${ }^{5}$ Propofol causes a propofol-mediated decrease in sympathetic activity, a decrease in systemic vascular resistance, and a decrease in cardiac output due to venous and arterial vasodilation, impaired baroreflex mechanism and depression of myocardial contractility. ${ }^{5-7}$ Even without the presence of cardiovascular disease, the incidence of this propofol-mediated hypotension ranges from $25 \%$ to $67.5 \% .^{8-10}$

Ephedrine has been widely used as a premedication in anesthesia for various operations; with a well-known role in the prevention of intraoperative hypotension especially during regional anesthesia. ${ }^{11}$ Many studies have concluded that the prophylactic injection of ephedrine could reduce the risk of hypotension by $14-37 \%$ when spinal anesthesia was used in pregnant patients for cesarean section. ${ }^{12-15}$

The most critical time for bradycardia and hypotension during anesthesia is immediately after induction and before tracheal intubation when the peak effect of induction drugs with minimal surgical stimulation.

In our study, the two groups show no differences in the base heart rate. The mean of the heart rate in CG in the first and second minutes show no difference between the two groups. The significant differences were observed in the $3 \mathrm{rd}$, 4th, and 5th minutes with P-value, $0.18,0.000,0.000$, respectively. However, no patient in the study participants had bradycardia.

Two groups show no differences in the base MAP. The mean of the heart rate in $\mathrm{CG}$ in the first minute shows no difference between the two groups. The significant differences were observed in the $2 \mathrm{nd}, 3 \mathrm{rd}$, 4th, and 5th minutes with P-value, 0.035, 0.000, 0.000 , and 0.000 , respectively.

No patient developed hypertension in the study groups. During the 1 st minute, no patient had hypotension. In the second minute, $12 \%$ of patients in the CG group developed hypotension compared to zero patients in EphG with no statistical difference (P-value 0.07). The percentage of patients in CG who developed hypotension in the $3 \mathrm{rd}$ and 4 th is $44 \%$ and $32 \%$ compared to $8 \%$ and $0 \%$ with significant differences with a p-value of 0.004 and 0.002 , respectively. No patient had hypotension in the 5 th minute.

Our findings are consistent with the study of El-Tahan in showing the effect of ephedrine in preventing hypotension induced by propofol and fentanyl. They studied patients undergoing valvular surgery. Doses of $0.07,0.1$, and $0.15 \mathrm{mg} / \mathrm{kg}$ of intravenous ephedrine were used. Unlike our study, their study included patients aged over 60 years and with ASA III and IV. ${ }^{11}$ This may explain the presence of significant side-effects such as tachycardia and subsequent risk of induced myocardial ischemia. ${ }^{11}$
The results of our study are also in line with the findings of Michelsen et al., who studied women above 60 years of age scheduled for minor gynecological surgeries. ${ }^{13}$ Ephedrine with doses of 0.1 and $0.2 \mathrm{mg} / \mathrm{kg}$, were used $1 \mathrm{~min}$ before induction of anesthesia with propofol and fentanyl. They found that both doses of ephedrine had prophylactic effects against hypotension. ${ }^{13}$

However, it's important to notice that the ephedrine effect may differ with different doses and other anesthesia drugs used with it. This was concluded in many studies. These were observed in the studies by Masjedi et al. and Gopalakrishna et al. ${ }^{15,16}$ Gopalakrishna et al. studied the effect of ephedrine on intubation and hemodynamic conditions during the rapid induction of anesthesia with propofol and rocuronium. They pre-treat their patients with doses of $0.075 \mathrm{mg} / \mathrm{kg}$ and $0.100 \mathrm{mg} / \mathrm{kg}$. They found that those doses improved the intubating conditions during rapid tracheal intubation but were not effective in preventing the hypotension following induction of anesthesia. ${ }^{16}$ Masjedi et al. observed the same when they used a dose of $0.070 \mathrm{mg} / \mathrm{kg}$. However, Masjedi et al. found that the use of $0.15 \mathrm{mg} / \mathrm{kg}$ ephedrine, i.e., was effective in controlling the hemodynamic parameters. This difference could be

Table 2. The heart rate between the two groups.

\begin{tabular}{lccc} 
Character & CG $(\mathrm{n}=25)$ & EphG $(\mathrm{n}=25)$ & P-value \\
Base HR & $82.63+8.3$ & $84.5+7.2$ & 0.3990 \\
1 & $86.3+11.2$ & $85.89+10.1$ & 0.8924 \\
\hline 2 & $83.3+11.6$ & $87.06+7.9$ & 0.1867 \\
3 & $79.4+10.8$ & $85.98+8.1$ & 0.0186 \\
\hline 4 & $74.8+9.9$ & $85.23+9.8$ & 0.0005 \\
5 & $74.6+10.9$ & $84.93+9.1$ & 0.0007 \\
\hline
\end{tabular}

$\mathrm{CG}=$ Control Group. $\mathrm{EphG}=$ Ephedrine. $\mathrm{HR}=$ Heart rate

Table 3. The mean arterial pressure between the two groups.

\begin{tabular}{lccc} 
Character & CG $(\mathrm{n}=25)$ & EphG $(\mathrm{n}=25)$ & P-value \\
Base MAP & $91.8+7.6$ & $90.77+10.1$ & 0.6855 \\
1 & $87.1+2.3$ & $88.3+5.8$ & 0.3411 \\
\hline 2 & $82.6+9.4$ & $87.2+5.0$ & 0.0358 \\
3 & $75.4+7.3$ & $83.7+9.0$ & 0.0008 \\
\hline 4 & $74.3+6.2$ & $82.7+6.0$ & 0.0001 \\
5 & $76.4+7.5$ & $87.6+6.4$ & 0.0001 \\
\hline
\end{tabular}

$\mathrm{CG}=$ Control Group. EphG = Ephedrine. MAP = Mean arterial pressure.

Table 4. The hypertension and hypotension.

\begin{tabular}{cccc} 
Character & CG $(\mathrm{n}=25)$ & EphG $(\mathrm{n}=25)$ & P-value \\
Hypertension & & & \\
1 & 0 & 0 & 1.000 \\
2 & 0 & 0 & 1.000 \\
3 & 0 & 0 & 1.000 \\
4 & 0 & 0 & 1.000 \\
5 & 0 & 0 & 1.000 \\
Hypotension & & 0 & \\
1 & 0 & 0 & 10.000 \\
2 & $3(12)$ & $2(8)$ & 0.0770 \\
3 & $11(44)$ & 0 & 0.0041 \\
4 & $8(32)$ & 0 & 0.0023 \\
5 & 0 & & 10.000 \\
\hline
\end{tabular}


explained by the higher potency of remifentanil used in Masjedi et al. study to cause bradycardia and hypotension in comparison with rocuronium. ${ }^{15}$

Gamlin et al. found that adding 15,20, or $25 \mathrm{mg}$ of ephedrine to $200 \mathrm{mg}$ of propofol in a 40 ASA 3 and 4 patients over 60 years, posted for genito-urinary surgery appeared to be effective to obtund the hypotensive response to propofol administration. However, marked tachycardia associated with the use of ephedrine in combination with propofol was observed in the majority of patients leading to their recommendation not to use the ephedrine/propofol mixtures in elderly patients. ${ }^{17}$

El-Beheiry et al. included an ASA class I-II, aged 21-60 years patients undergoing elective outpatient surgery. The patients received pre-induction ephedrine sulphate of a dose of 0.070 $\mathrm{mg} / \mathrm{kg}$, pre-induction volume loading of $12 \mathrm{~mL} / \mathrm{kg}$ Ringer's lactate, or no treatment. Rapid-sequence intubation with cricoid pressure using succinylcholine was used. Their findings showed that pre-induction administration of ephedrine sulphate is less efficacious than preoperative volume loading in maintaining hemodynamic stability during rapid-sequence induction with propofol and succinylcholine. ${ }^{18}$

Agarwal et al. also concluded that preoperative administration of ephedrine failed to prevent propofol-induced hypotension, but preoperative volume loading with $10 \mathrm{~mL} / \mathrm{kg}$ of ringer lactate successfully antagonized it. ${ }^{19}$ Malla et al. conclude that crystalloid preloading is not efficacious in preventing hypotension compared to gelatine and ephedrine which were markedly attenuated, but does not fully abolish the decrease in blood pressure caused by induction. ${ }^{20}$

Dhungana et al. found that preloading with colloid (Haemaccel, $10 \mathrm{~mL} / \mathrm{kg}$ intravenously over $10-15$ minutes) or prior injection of sympathomimetic ephedrine $(0.2 \mathrm{mg} / \mathrm{kg} i v)$, prior to induction of anesthesia with propofol $(2.5 \mathrm{mg} / \mathrm{kg} \mathrm{iv})$ though are not fully efficacious in preventing hypotension caused by propofol induction, both decrease the incidence in a significant number of patients with heart rate less than baseline value in the colloid group. ${ }^{21}$

\section{Conclusions}

The use of small doses of ephedrine could attenuate the propofol-fentanyl hypotensive effect during the induction of general anesthesia without tachycardia.

\section{References}

1. White P. Propofol: Its Role in Changing the Practice of Anesthesia. Anesthesiol 2008;109:1132-1136.

2. Tobias J, Leder M. Procedural sedation: A review of sedative agents, monitoring, and management of complications. Saudi J Anaesth 2011;5:395.

3. Hussein MM, Mostafa RH, Ibrahim IM. Intravenous ephedrine, phenylephrine, and ketamine for attenuation of hypotension associated with induction of general anesthesia with propofol. Ain-Shams J Anaesthesiol 2017;10:182.

4. Ma G, Bavadekar SA, Davis YM, et al. Pharmacological effects of ephedrine alkaloids on human alpha(1)- and alpha(2)-adrenergic receptor subtypes. J Pharmacol Exp Ther
2007;322:214-21.

5. Smith C, McEwan AI, Jhaveri R et al. The interaction of fentanyl on the $\mathrm{Cp} 50$ of propofol for loss of consciousness and skin incision. Anesthesiol 1994;81:820-8.

6. Lysakowski C, Dumont L, Pellegrini M et al. Effects of fentanyl, alfentanil, remifentanil and sufentanil on loss of consciousness and bispectral index during propofol induction of anaesthesia. Br J Anaesth 2001;86:523-7.

7. Claeys MA, Gepts E, Camu F. Haemodynamic changes during anaesthesia induced and maintained with propofol. $\mathrm{Br} \mathrm{J}$ Anaesth 1988;60:3-9.

8. Smith I, White PF, Nathanson M, Gouldson R. Propofol. An update on its clinical use. Anesthesiol 1994;81:1005-43.

9. Bano F, Zafar S, Sabbar S, et al. Intravenous ketamine attenuates injection pain and arterial pressure changes during the induction of anesthesia with propofol: A comparison with lidocaine. J Coll Physicians Surg Pak 2007;17:390-3.

10. Larsen R, Rathgeber J, Bagdahn A, et al. Effects of propofol on cardiovascular dynamics and coronary blood flow in geriatric patients. A comparison with etomidate. Anaesthesia 1988;43:25-31.

11. El-Tahan MR. Preoperative ephedrine counters hypotension with propofol anesthesia during valve surgery: A dose dependent study. Ann Card Anaesth 2011;14:30-40.

12. Kasaba T, Yamaga M, Iwasaki T, et al. Ephedrine, dopamine, or dobutamine to treat hypotension with propofol during epidural anesthesia. Can J Anaesth 2000;47:237-41.

13. Michelsen I, Helbo-Hansen HS, Køhler F et al. Prophylactic ephedrine attenuates the hemodynamic response to propofol in elderly female patients. Anesth Analg 1998;86:477-81.

14. Ozkoçak I, Altunkaya H, Ozer Y et al. Comparison of ephedrine and ketamine in prevention of injection pain and hypotension due to propofol induction. Eur J Anaesthesiol 2005;22:44-8.

15. Masjedi M, Zand F, Kazemi AP, Hoseinipour A. Prophylactic effect of ephedrine to reduce hemodynamic changes associated with anesthesia induction with propofol and remifentanil. J Anaesthesiol Clin Pharmacol 2014;30:217-21.

16. Gopalakrishna MD, Krishna HM, Shenoy UK. The effect of ephedrine on intubating conditions and haemodynamics during rapid tracheal intubation using propofol and rocuronium. Br J Anaesth 2007;99:191-4.

17. Gamlin F, Vucevic M, Winslow L, Berridge J. The haemodynamic effects of propofol in combination with ephedrine. Anaesthesia 1996;51:488-491

18. El-Beheiry H, Kim J, Milne B, Seegobin R. Prophylaxis against the systemic hypotension induced by propofol during rapid-sequence intubation. Can J Anaesth 1995;42:875-8

19. Agarwal A, Sharma K, Parashar S, et al. Prevention of hypotension during propofol induction: a comparison of preloading with Ringer lactate and intravenous ephedrine. J Evol Med Dent Sci 2013;2:6640-50

20. Malla S, Andrabi R, Ashraf S, Saleem B. Prevention of hypotension caused by induction of anaesthesia with propofol, a comparison of preloading with $3.5 \%$ polymers of degraded gelatin solution, crystalloid (Ringer lactate) \& intravenous ephedrine. J Evol Med Dent Sci 2015;4:365-74.

21. Dhungana Y, Bhattarai BK, Bhadani UK et al. Prevention of hypotension during propofol induction: a comparison for preloading with $3.5 \%$ polymers of degraded gelatin (Haemaccel) and intravenous ephedrine. Nepal Med Coll J 2008;10:16-19. 